\title{
An active RNA transport mechanism into plant vacuoles
}

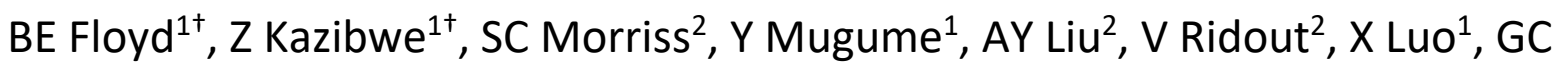 Maclntosh $^{2 *}$, DC Bassham ${ }^{1 *}$}

1 Department of Genetics, Development and Cell Biology, lowa State University, Ames, IA

2 Roy J. Carver Department of Biochemistry, Biophysics and Molecular Biology, lowa State University, Ames, IA

† These authors contributed equally to this work

*Corresponding authors:

Diane C. Bassham: bassham@iastate.edu

Gustavo C. MacIntosh: gustavo@iastate.edu 
bioRxiv preprint doi: https://doi.org/10.1101/2021.07.28.454214; this version posted July 28, 2021. The copyright holder for this preprint (which

was not certified by peer review) is the author/funder, who has granted bioRxiv a license to display the preprint in perpetuity. It is made available under aCC-BY-NC-ND 4.0 International license.

\begin{abstract}
RNA degradation inside the plant vacuole by the ribonuclease RNS2 is essential for maintaining nucleotide concentrations and cellular homeostasis via the nucleotide salvage pathway. However, the mechanisms by which RNA is transported into the vacuole are not well understood. While selective macroautophagy may contribute to this transport, macroautophagy-independent transport pathways also exist. Here we demonstrate a mechanism for direct RNA transport into vacuoles that is active in purified vacuoles and is ATP hydrolysis-dependent. We identify the RNA helicase SKI2 as a factor required for this transport pathway, as ski2 mutant vacuoles are defective in transport. ski2 mutants have an increased autophagy phenotype that can be rescued by exogenous addition of nucleosides, consistent with a function in nucleotide salvage. This newly-described transport mechanism is therefore critical for RNA degradation, recycling and cytoplasmic nucleotide homeostasis.
\end{abstract}




\section{Introduction}

Nucleotide salvage, the recycling of nucleosides and nucleobases to produce nucleotides, is necessary to maintain cellular homeostasis in eukaryotes ${ }^{1-3}$. Cells have the ability to produce nucleotides through de novo synthesis or through the salvage pathway, but when nucleosides or nucleobases are available, the salvage pathway is preferred due to its favorable energy balance ${ }^{4}$. However, the salvage pathway is not simply a complement to the de novo biosynthetic pathway. The salvage pathway removes bases and nucleosides that can have an inhibitory effect on metabolism, and in plants it is also linked to production of cytokinins ${ }^{1}$.

The main source of nucleosides for nucleotide salvage is thought to be RNA degradation ${ }^{5}$, and given its abundance, ribosomal RNA is likely the most prevalent source. Previous work showed that rRNA is degraded by RNS2, the main ribonuclease in the Arabidopsis vacuole ${ }^{6}$. Mutant plants lacking RNS2 activity, or plants expressing a mutant version of the protein that is mislocalized outside the vacuole, accumulate rRNA. These mutants also have constitutive activation of autophagy, a process usually only highly active during stress ${ }^{6-8}$. Transcriptional and metabolic analyses suggested that plants lacking vacuolar RNA turnover compensate by activation of the pentose-phosphate pathway, which provides ribose-5-P to the de novo purine biosynthesis pathway ${ }^{9}$. Moreover, supplementing rns 2 mutant plants with purine nucleosides (inosine or hypoxanthine) suppresses their constitutive autophagy phenotype, reinforcing the idea that defective RNA recycling leads to a decrease in purine availability that triggers autophagy as a compensatory mechanism ${ }^{10}$. Genetic analyses indicated that some components of the autophagy machinery are necessary for efficient transport of rRNA from the cytoplasm to the vacuole ${ }^{8}$, suggesting that a selective autophagy mechanism similar to yeast ribophagy ${ }^{11}$ may exist in plants. However, rRNA transport into the vacuole was unaffected in rns2-2 atg9-4 mutants, indicating that a macroautophagy-independent transport mechanism may also participate in nucleic acid import into the Arabidopsis vacuole ${ }^{8}$.

An alternative pathway for RNA transport into lytic organelles, called RNautophagy, has been described for animal cells ${ }^{12}$. In RNautophagy, RNA is transported into lysosomes by a direct import mechanism that requires at least two lysosomal proteins, LAMP2C and SIDT2 ${ }^{12,13}$; however, these proteins have not been described in plants. This transport mechanism is capable of transporting rRNA and requires ATP ${ }^{12}$. RNautophagy seems to have a significant contribution to the degradation of cellular RNAs ${ }^{12,13}$. We hypothesized that an analogous RNA transport mechanism could be present in Arabidopsis and mediate the macroautophagy-independent import of rRNA into vacuoles observed in autophagy defective mutants.

In this study, we developed assays to measure the transport of rRNA and a synthetic fluorescent RNA into isolated vacuoles. Using these methods, we demonstrated the presence in Arabidopsis of an ATPdependent novel transport mechanism that can carry RNA into the vacuole. We also identified an RNA helicase, SKI2, that is necessary for this vacuolar transport mechanism. Our results demonstrate that plants have a mechanism analogous to RNautophagy, but requiring distinct machinery, and provide tools for the dissection of this novel mechanism that can contribute to nucleotide salvage and cellular homeostasis.

\section{Results}

Isolated Arabidopsis vacuoles can import exogenous rRNA 
A Blastp search ${ }^{14}$ of the predicted proteomes of Arabidopsis and 63 other plant species in the Phytozome database ${ }^{15}$ using human and mouse SIDT2 and LAMP2C as queries did not return any significant hits. If RNA can be transported directly across the vacuolar membrane in plants, it must therefore occur via an unrelated mechanism. To test whether an autonomous vacuolar transport mechanism exists in Arabidopsis, independent of SIDT2 and LAMP2C-like proteins, we set up an in vitro system to measure direct rRNA uptake using isolated vacuoles. A challenge with quantification of RNA incorporation into vacuoles is the presence of RNS2, the main vacuolar RNase in Arabidopsis ${ }^{6,7}$, which would degrade any RNA that is imported into the organelle. To avoid this problem, we isolated vacuoles from rns2-2 null mutant plants, which normally accumulate RNA due to reduced degradation ${ }^{6,8}$. To distinguish between Arabidopsis RNA already present in rns2-2 vacuoles and RNA transported into the vacuole during the assay, we used Drosophila RNA as a transport substrate instead of plant RNA. A schematic of the experimental design is shown in Figure 1A. Purified vacuoles were incubated with $5 \mu \mathrm{g}$ total Drosophila RNA in either the presence or absence of ATP. The samples were gently mixed and incubated for 5 minutes at room temperature to allow transport. To prevent contamination of minus ATP samples with cellular ATP released during vacuole purification, apyrase was added to the samples without exogenously added ATP. After allowing transport to occur, RNase A was added for 20 minutes on ice to remove RNA not incorporated into vacuoles; Drosophila RNA taken up by vacuoles would be protected from RNase A degradation. Following addition of an RNase A inhibitor, RNA was extracted from the vacuoles and quantified by qRT-PCR using Drosophila rRNA specific primers (Extended Figure 1). Vacuolar acid phosphatase activity was used for normalization as in Floyd et at ${ }^{8}$. Using this assay, we were able to detect Drosophila rRNA uptake into plant vacuoles. Vacuoles incubated with ATP accumulated $\sim 3$-fold more $18 \mathrm{~S}$ and $28 \mathrm{~S}$ Drosophila rRNA than those without ATP (Figure 1B). Control samples lacking exogenous RNA showed no accumulation of rRNA, indicating that the Drosophila rRNA primers can discriminate between Drosophila rRNA and the Arabidopsis rRNA already present in the vacuole. Control samples lacking purified vacuoles show the quantity of detectable RNA remaining after RNase A treatment (Figure 1B). These results indicated that vacuoles purified from rns2-2 plants can import RNA in an ATP-dependent manner, analogous to the RNautophagy mechanism reported in mammals ${ }^{12}$.

\section{Fluorescent synthetic RNA can be used to assay vacuolar trans-membrane transport activity}

To confirm the results of RNA transport identified by qRT-PCR analysis, an assay was developed to more directly visualize RNA transport into purified vacuoles using fluorescent RNA (FL-RNA). We used a commercially synthesized 15-nucleotide random RNA sequence conjugated at the 3'-end with Alexa488 fluorophore as transport cargo. A schematic of the experimental design is shown in Figure $2 \mathrm{~A}$. Wild type vacuoles were used in this analysis since intravacuolar RNA degradation should not interfere with the quantification of fluorescence inside the vacuole. Purified vacuoles were incubated for 20 minutes with FL-RNA in the presence of ATP to promote transport; room temperature incubation was found to be unnecessary as measurable transport occurred on ice, under which conditions stability of the vacuoles is improved. Following FL-RNA transport incubation, samples were diluted ten-fold with vacuole buffer to dilute out non-imported FL-RNA, reducing background fluorescence. Vacuoles were then imaged using microscopy. Using this assay, we were able to observe fluorescence accumulation inside isolated Arabidopsis vacuoles (Figure 2 B-D). We observed that two classes of fluorescent vacuole were found, exhibiting either low or high fluorescence (Figure 2B).

To determine if the observed fluorescence was inside the vacuole or binding to the outer surface, vacuoles were imaged using confocal microscopy. Vacuoles with either low or high fluorescence were imaged from multiple focal z-layers and for both high and low fluorescence vacuoles fluorescence was 
found throughout the organelle at multiple z-positions rather than the outer edges alone (Figure 2C). The fluorescent RNA is therefore within the lumen of the vacuole rather than bound to the membrane. These observations indicate that FL-RNA can be used to visualize the RNautophagy-like transport pathway in purified Arabidopsis vacuoles, and that the transport mechanism can transport small RNAs in addition to rRNA.

\section{The efficient transport of FL-RNA into vacuoles needs ATP hydrolysis}

After determining that the observed fluorescence was originating from the vacuole interior for both low and high fluorescence vacuoles, we assessed the requirements for transport into the isolated wild-type vacuoles. First, we determined the percentage of vacuoles observed to be fluorescent in the presence or absence of ATP. About $20 \%$ of vacuoles showed a fluorescence signal regardless of treatment (Figure 2D), with the remainder being non-fluorescent. Vacuoles alone, without addition of FL-RNA, showed no autofluorescence. This suggests that only a subpopulation of vacuoles is competent for transport, although the reason for this is unknown.

We then determined the distribution of vacuole fluorescence intensities in the FL-RNA transport system. Vacuoles were manually assigned to different categories, high FL, low FL, and zero FL (Figure 2B and 2E). Vacuoles showing a fluorescence intensity that saturated or nearly saturated the detector were assigned to the high-FL group. Vacuoles showing all other amounts of fluorescence were assigned to the low-FL group. Vacuoles showing no fluorescence were assigned to the zero-FL group. Using this ranking for quantification, there was a significant increase in vacuoles in the high FL population when ATP was added (Figure 2E), suggesting that efficient transport requires ATP. We confirmed that the observed transport of the fluorophore into the vacuoles required the presence of the RNA, and that the Alexa488 fluorophore alone was unable to enter the vacuoles by a non-selective process. For this, the FL-RNA probe was incubated with RNase A to digest the RNA portion of the probe prior to incubation with vacuoles and ATP. RNase A treatment of the FL-RNA probe resulted in few high-FL vacuoles, indicating that RNA enhances transport of the Alexa488 fluorophore (Figure 2E). The number of low FL vacuoles was similar in the minus ATP samples and in the samples with probe pre-treated with RNase A (Figure $2 \mathrm{D}$ and $2 \mathrm{E}$ ). These results suggest that the low $\mathrm{FL}$ vacuoles represent non-selective transport that is independent of RNA and ATP, while high FL vacuoles represent an ATP-dependent RNA transport system. Identical results were obtained using vacuoles purified from rns2-2 plants (Extended Figure 2), indicating that the same RNA-transport mechanism is functional in the wild-type and mutant backgrounds.

To eliminate bias in our analysis, we used Fiji software ${ }^{16}$ to measure the fluorescence intensity of individual fluorescing vacuoles. A circular region within the vacuole being measured was quantified, and average intensities for each sample were statistically compared (Figure 3). Wild type vacuoles treated with ATP showed the highest fluorescence intensity, as well as the widest distribution of fluorescence intensities. Removal of ATP resulted in overall decreased fluorescence and a narrower distribution (Figure 3A). Although bright vacuoles were seen in minus ATP and in the RNase A-treated probe samples, they were mostly statistical outliers following analysis (vacuole $\mathrm{FL}>1.5$ standard deviations away from sample mean). Background fluorescence was quantified to illustrate the level of fluorescence from non-transported FL-RNA remaining after sample dilution. Vacuoles alone showed no quantifiable autofluorescence. Similar results were obtained for rns2-2 vacuoles (Figure 3B).

To further elucidate the requirements of the vacuolar RNA transport mechanism, we tested the effect of inhibitors on FL-RNA transport. We first tested whether ATP hydrolysis was required or whether ATP 
binding alone was sufficient for RNA transport. The addition of adenosine $5^{\prime}$-( $\beta, \gamma$-imido) triphosphate to the vacuole transport assay, which contains a non-hydrolysable bond between the $\beta$ and $\gamma$ phosphates, significantly reduced the mean vacuole fluorescence and lowered the fluorescence distribution (Figure 4A). This indicates that ATP hydrolysis was required for efficient transport. A common ATP-dependent mechanism of trans-membrane transport in many organisms involves ATP-binding cassette (ABC) transporter proteins ${ }^{17}$. Treatment of vacuoles with the $A B C$ transporter inhibitor sodium orthovanadate, a suppressor of ATPase activity ${ }^{18}$, had no significant effect on FL-RNA transport efficiency (Figure 4B), suggesting that RNA transport into the vacuole does not involve an $A B C$ transporter. Co-transporters are another class of proteins used in the trans-membrane transport of molecules into the vacuole ${ }^{19}$. Plant vacuoles maintain a high concentration of ions in their lumen that drive antiport mechanisms ${ }^{19}$. To test whether FL-RNA transport relies on the ion gradient in plant vacuoles, we treated vacuoles with gramicidin to depolarize the membrane ionic gradient ${ }^{20,21}$. Treatment of vacuoles with gramicidin had no significant effect on FL-RNA transport efficiency (Figure $4 C$ ), indicating that RNA transport into the vacuole does not require an ion gradient to drive transport.

\section{SKI2 is necessary for FL-RNA transport into vacuoles}

As a first attempt to identify proteins involved in this vacuolar RNA transport mechanism, we used a candidate gene approach. We searched the published tonoplast proteome of Arabidopsis vacuoles ${ }^{22}$ for proteins with RNA-binding motifs. In this proteome dataset we identified two proteins annotated as RNA helicases (encoded by At3g06480 and At3g46960) as the top candidates, as they possessed RNAbinding domains and ATP hydrolysis activity. At3g46960 corresponds to SUPERKILLER 2 (AtSKI2), the gene encoding for a DExD/H box RNA helicase associated with the cytoplasmic RNA exosome ${ }^{23,24}$; while At3g06480 corresponds to RNA Helicase 40 (RH4O), encoding a DEAD box RNA helicase with homology to helicases involved in rRNA processing ${ }^{25,26}$. To determine if either of these proteins had a role in the vacuolar RNA transport mechanism, we obtained T-DNA insertion Arabidopsis lines (termed ski2-5, rh401 and $r$ 40-2) that disrupted the corresponding genes and identified individual plants homozygous for each mutation, which lacked expression of the corresponding target gene (Extended Figure 3 ). Then, we isolated vacuoles from the ski2 and rh40 mutant lines and tested their ability to transport RNA using the FL-RNA system.

Vacuoles isolated from the ski2-5 mutant plants had significantly reduced FL-RNA transport capability, and this vacuolar RNA transport was restored to wild-type levels after complementation of the ski2 mutant with an extra copy of the SKI2 gene (Figure 5A and Extended Figure 3). On the other hand, vacuoles from two independent $r h 40$ mutants had normal FL-RNA transport levels (Figure 5B). These results indicated that SKI2 is necessary for the Arabidopsis RNautophagy-like mechanism, while RH40 is not.

Vacuolar RNA degradation is critical for the maintenance of nucleotide homeostasis ${ }^{9,10}$. Mutants with deficiencies in the nucleotide salvage pathway, including those with defects in vacuolar RNA degradation such as $r n s 2^{6-8}$, have constitutive activation of the autophagy pathway. We therefore tested whether these RNA helicase mutants have constitutive autophagy, indicating a physiological role in maintaining nucleotide concentrations. Initially we used monodansylcadaverine (MDC) staining to identify acidic organelles that could correspond to autophagosomes (Extended Figure 4). This analysis showed an increase in MDC-positive puncta in ski2 mutant cells and, unexpectedly, also in the rh40 mutants. Complementation of the ski2 line with a wild-type copy of the $S K I 2$ gene resulted in complete reversal of the autophagy phenotype. To confirm that the increase in MDC puncta corresponded to an increase in autophagosomes, protoplasts from wild type and ski2-5 mutant plants were transiently 
transformed with the autophagy reporter GFP-AtATG8 $e^{8}$. Microscopy analysis of these protoplasts showed that ATG8-labeled autophagic structures accumulate in the ski2-5 line at levels similar to those observed in the rns 2 mutants, while complementation with an extra copy of SKI2 restores basal autophagy to wild type levels (Figure 6A and B). An increase in flux through the autophagy pathway in ski2-5 mutant seedlings was confirmed by immunoblotting with ATG8 antibodies in the presence or absence of concanamycin A to block vacuolar degradation. As ATG8 is degraded during autophagy, the accumulation of ATG8 protein is a proxy for the activity of the autophagy pathway ${ }^{27}$. An increase in ATG8 was seen in the ski2-5 mutant, and this increase was rescued in the complemented line (Figure 6CF).

Constitutive autophagy has been observed for rns2 mutants, which are defective in the vacuolar RNA salvage pathway, but disturbance in other pathways that affect cellular homeostasis can also trigger an autophagy phenotype ${ }^{28}$. However, the increase in autophagy observed in rns 2 lines can be reversed by incubation in media containing purines, supporting the role of the RNase in nucleotide homeostasis maintenance ${ }^{10}$. Thus, to test whether SKI2 and RH40 participate in the RNA salvage pathway, we used inosine treatments to chemically complement the cellular phenotype. The addition of this purine to the growth medium was able to reverse the ski2-5 autophagy phenotype (Figure 6; Extended Figure 5 and 6 ) but failed to revert the phenotype of the $r h 40$ mutant (Extended Figure 5 and 7), consistent with the lack of effect on RNA transport in this mutant (Figure 5B). The amount of inosine needed to revert the ski2-5 autophagy phenotype was higher than the amount needed for reversion of the rns 2 autophagy phenotype in seedlings. However, the autophagy phenotype in protoplasts of both ski2-5 and rns2-2 (Extended Figure 6) was reversed at a lower inosine concentration, probably because protoplasts can take up inosine more easily compared to seedlings.

While the observed phenotypes strongly support a role for SKI2 in the RNA salvage pathway, RH40 does not seem to function in the vacuolar RNA transport or the RNA salvage pathways. Although RH4O has not been characterized in Arabidopsis, it has homology to RNA helicases involved in ribosome processing 25. Thus, the constitutive autophagy phenotype observed for the $r h 40$ mutant could be related to defects in this process. Defects in rRNA processing lead to nucleolar stress, which, in animals, induces autophagy 29 .

\section{Discussion}

The vacuole RNA salvage pathway is an important component of nucleotide homeostasis in plants ${ }^{30}$. While the main vacuolar RNase involved in this process has been well-characterized ${ }^{6,7,31}$, the mechanisms that translocate RNA from the cytoplasm to the vacuole are less understood. Here, we established a straightforward assay to study RNA transport and identified a novel mechanism present in Arabidopsis vacuoles. This vacuolar RNA import system seems functionally analogous to the RNautophagy mechanism described in animals. Animal RNautophagy can transport rRNA and short oligonucleotides ${ }^{12,32}$ through an ATP-dependent process that functions in isolated lysosomes ${ }^{12}$. We found that, in a similar fashion, isolated vacuoles can transport rRNA and short oligonucleotides in an ATP-dependent manner. Moreover, we showed that ATP hydrolysis is necessary for optimal transport and that neither $A B C$ transporters nor a proton gradient are involved in this process. Interestingly, mutant mice defective in the RNautophagy process have an induced muscular dystrophy-like phenotype, and skeletal muscle fiber cells show an increase in autophagolysosomes ${ }^{33}$, and Arabidopsis mutants defective in vacuolar RNA transport show an increase in autophagy. However, the plant and animal systems cannot be mediated by the same mechanism. Animal RNautophagy requires two lysosomal proteins, Lysosome-associated membrane glycoprotein 2 isoform C (LAMP2C) and SID1 transmembrane 
family member 2 (SIDT2) ${ }^{12,13}$. These proteins function synergistically to bind and transport RNA ${ }^{34}$. Both proteins localize to the lysosome membrane ${ }^{13,35}$ and contain arginine-rich RNA binding motives in their cytosolic domains and show preference for RNA with poly(G) sequences ${ }^{32,34,36}$. However, genes encoding LAMP2C and SIDT2 are absent from plant genomes ${ }^{30}$, and therefore the pathway we have discovered in Arabidopsis must use a distinct protein machinery. Thus, the plant vacuolar RNA transport uses a different, previously undescribed, mechanism for RNA import into the lytic organelle. While it has been proposed that the animal RNautophagy involves transport through a channel-type mechanism, we cannot discard alternative mechanisms in plants. For example, the transport observed in our experiments could be a type of microautophagy, a process that uses different mechanisms involving invagination of the vacuole membrane ${ }^{37}$. Although less well-characterized, the presence of microautophagy processes in plants has been reported ${ }^{38}$.

Our candidate gene approach identified SKI2 as a putative component of the transport system. Vacuoles from ski2 mutant plants have compromised RNA transport and show the same constitutive autophagy phenotype as rns2 mutants that lack the main vacuolar RNase activity ${ }^{6,8}$. Moreover, altered nucleotide homeostasis in mutants with defective vacuolar RNA salvage can be compensated by feeding seedlings with purine nucleosides, and the rns 2 constitutive autophagy phenotype is chemically complemented by inosine feeding in a dose-dependent manner ${ }^{10}$. Consistent with a role in the RNA salvage pathway, the ski2 autophagy phenotype is also complemented by inosine feeding, although the concentration of inosine needed for this complementation is higher than the concentration needed to eliminate the rns 2 phenotype. While RNS2 contributes to nucleoside homeostasis through the vacuolar RNA salvage pathway, SKI2 may contribute to nucleotide homeostasis through both the vacuolar and cytoplasmic RNA salvage pathways. We show here that SKI2 is necessary for the RNautophagy-like mechanism that delivers RNA to the vacuole for degradation. In addition, as part of the RNA exosome ${ }^{23,24}$, SKI2 mediates several processes that involve cytoplasmic degradation of cellular RNAs, including normal mRNA decay 24,39 , and quality control mechanisms such as nonsense mediate decay ${ }^{40,41}$, no-go decay ${ }^{42}$, nonstop decay ${ }^{43}$, and nonfunctional rRNA decay ${ }^{44}$. Thus, the ski2 mutant may present a more severe deficiency in the salvage pathway that requires higher concentration of purine in the growth medium to achieve normal levels. A role in nucleotide homeostasis for RNA exosome-mediated RNA decay has also been proposed in animals ${ }^{45}$.

The detection of SKI2 in the vacuole proteome ${ }^{22}$ and the absence of other members of the SKI complex in this protein fraction, the ATP requirement for vacuolar RNA transport, and the ski2 phenotypes described here, support the hypothesis that SKI2 is a component of a plant RNautophagy-like mechanism. Alternatively, an indirect effect could be invoked. The cytoplasmic RNA exosome and the SKI complex have an important role in the degradation of mRNA decay intermediates. If $5^{\prime}$ fragments produced by endonucleolytic cleavage of abundant transcripts are not removed by the cytoplasmic RNA exosome, they can be recognized as aberrant transcripts by the cellular PTGS machinery, triggering silencing of the corresponding mRNA ${ }^{46-48}$. Plants with knock-down of core components of the exosome can have pleiotropic phenotypes, such as altered cuticular wax composition, due to the silencing of genes associated with these pathways ${ }^{49,50}$. Thus, it is possible that the ski2 mutant is missing a key component of the RNA transport system due to defects in RNA exosome function suppressing silencing of endogenous transcripts.

In conclusion, plant vacuoles have the ability to actively import RNA through a mechanism that is functionally analogous to RNautophagy, but mechanistically distinct. The RNA helicase SKI2 is needed for a fully active mechanism and it is likely to be a component of the transport system. This novel mechanism can translocate rRNA and thus may have a significant role in RNA salvage and nucleotide 
homeostasis in plant cells, and its ability to transport other RNAs, represented by random sequence short oligonucleotides in our assay, could indicate that this mechanism is also important in the turnover of other cytoplasmic RNA species. The assays developed for this work should facilitate the molecular dissection of this new mechanism.

\section{Methods}

Plant growth and Arabidopsis thaliana genotypes. Arabidopsis thaliana plants used for vacuole analyses were grown in soil under short day conditions ( $10 \mathrm{hr}$ light $/ 14 \mathrm{hr}$ dark) at $22^{\circ} \mathrm{C}$ for 6 weeks. For whole seedling microscopy, seeds were surface-sterilized in 33\% (v/v) bleach, $1 \%$ Triton X-100 for 20min followed by cold treatment for $\geq 2$ days. Plants were grown for seven days under long day (LD) conditions (16hr light/8hr dark) at $22^{\circ} \mathrm{C}$ on nutrient solid half-strength Murashige-Skoog (MS) medium with vitamins (MSP09; Caisson Labs), 1\% sucrose, 2.4mM MES pH5.7, and 0.8\% (w/v) phytoagar (PTP01; Caisson Labs) as described ${ }^{51}$. Arabidopsis Columbia- 0 accession was used as wild type control. T-DNA insertion mutants used in this study were $r n s 2-{ }^{6}{ }^{6}$, ski2-5 (SALK_141579C), rh40-1 (CS824414) and rh402 (SALK_054998). To generate the ski2-5/SKI2 line, The SKI2 cDNA sequence was amplified from wild type cDNA using CloneAmp HiFi PCR Premix (Takara) and cloned into the PPZP212 binary vector, resulting in a SKI2 gene driven by 35S promoter at the $5^{\prime}$ end, and with a MYC tag sequence at the $3^{\prime}$ end of the insert. Transgenic plants were generated by the floral dip method. Genotyping was carried out using standard qPCR and PCR techniques. Mutants and transgenic lines are available from the corresponding authors upon request. A list of primers used in this work is provided in Supplementary Table 1.

qRT-PCR-based assay of in vitro RNA transport into vacuoles. Vacuoles were purified from rns2-2 plants and membrane integrity confirmed by neutral red staining and brightfield microscopy according to Robert et al. ${ }^{52}$. Two vacuole preparations were used for each experiment. Purified vacuoles were combined into one tube and gently homogenized by pipetting with cut tips. A $200 \mu$ l aliquot of vacuole preparation was added to each reaction tube. $5 \mu \mathrm{l}$ of $2 \mathrm{U} / \mu \mathrm{l}$ apyrase (A6410; Sigma Aldrich) was added to minus ATP samples and incubated for 5 minutes at room temperature to remove residual ATP. $5 \mu \mathrm{g}$ Drosophila melanogaster total RNA (purified as previously described ${ }^{53}$ ) were dissolved in nuclease free water, then added to the reaction along with $18 \mathrm{mM}$ adenosine $5^{\prime}$-triphosphate (ATP) magnesium salt (A9187; Sigma Aldrich) for plus ATP samples. Following gentle mixing with cut pipet tips, samples were incubated at room temperature for 5 minutes to allow transport. Samples were then incubated with 200 $\mathrm{gg}$ RNaseA (19101; Qiagen) for 20min on ice to remove residual Drosophila RNA outside the vacuole. $10 \mu \mathrm{l}$ Recombinant RNasein (C) Ribonuclease inhibitor (N2515; Promega) was added to inhibit RNaseA activity and samples were flash frozen in liquid nitrogen until analyzed. Upon thawing for analysis, $18 \mathrm{mM}$ ATP was added to samples without prior ATP addition, as the ATP concentration affects acid phosphatase activity. Vacuoles were homogenized by vortexing and spun down prior to aliquoting $35 \mu \mathrm{l}$ for use in acid phosphatase analysis as described ${ }^{8}$. RNA was extracted from the remaining vacuoles using a RNeasy Plant Mini Kit (74904; Qiagen). For this, vacuoles were combined with $600 \mu$ l of lysis buffer RLT (Qiagen) and RNA extracted according to the manufacturer's protocol. Samples were DNase-

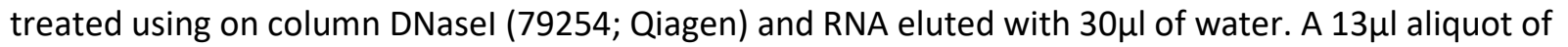
extracted vacuolar RNA was used for cDNA synthesis by a qScript Flex cDNA kit (95049; Quanta BioSciences) using random primers with a final CDNA dilution volume of $80 \mu \mathrm{l}$. The quality of cDNA was tested by semi-quantitative PCR. qRT-PCR was carried out using PerfeCta SYBR Green Fast Mix, Low ROX (95074; Quanta BioSciences) in a Stratagene Mx4000 multiplex quantitative PCR system (Agilent Technologies) using primers for Drosophila $18 \mathrm{~S}$ and $28 \mathrm{~S}$ rRNA. qPCR results were normalized to the vacuole marker enzyme acid phosphatase activity ${ }^{54,55}$. Samples not containing vacuoles were assigned 
an acid phosphatase value based on each experiment's average acid phosphatase activity for normalization purposes.

Visualizing RNA transport into the vacuole using Alexa Fluor $\mathbf{4 8 8}$ labeled RNA. Vacuoles were purified from wild type and mutant ( $r n s 2-2$, ski2-5, rh40-1, rh40-2) rosette leaves. 200 $\mu$ l purified vacuoles were used for each sample following gentle sample homogenization by pipetting, and kept on ice. Vacuoles were treated with apyrase and $10 \mathrm{mM}$ ATP. $1 \mu \mathrm{l}$ of $413 \mathrm{pmol} / \mu \mathrm{l}$ Alexa488-RNA was added. The Alexa488RNA was a custom machine mixed random 15 base ribonucleotide sequence with an Alexa488 molecule conjugated to the $3^{\prime}$ end of the oligo and HPLC purified (Integrated DNA technologies). As a negative control, Alexa488-RNA was incubated with 50 $\mu$ g of RNaseA (19101; Qiagen) for 20-30 minutes at room temperature in darkness to remove RNA from the Alexa488 fluorophore. Prior to visualization by fluorescence microscopy the samples were diluted 10 -fold with ice-cold vacuole buffer (Robert et al. 2007) to dilute residual Alexa488 fluorophore. Vacuoles were then imaged by fluorescence or confocal microscopy. For inhibitor studies, $200 \mu$ l purified wild-type vacuoles were prepared as above. Vacuoles were treated for 5 minutes on ice with either $10 \mathrm{mM}$ adenosine $5^{\prime}-(\beta, \gamma$-imido) triphosphate lithium salt hydrate (A2647; Sigma Aldrich), 15 $\mu$ M gramicidin (G5002; Sigma Aldrich), or sodium orthovanadate (S6508; Sigma Aldrich). Following incubation with either orthovanadate or gramicidin,10mM ATP and $1 \mu \mathrm{l}$ of $413 \mathrm{pmol} / \mu \mathrm{l}$ Alexa488-RNA was added to samples to measure vacuole RNA transport. Samples were incubated on ice for $20 \mathrm{mins}$, diluted and visualized by confocal or fluorescence microscopy.

Microscopy for RNA transport and autophagy quantification. Vacuoles were imaged by confocal microscopy using a NikonC1si confocal scanning system attached to a 90i microscope (Nikon Instruments). For quantification experiments, vacuoles were visualized using a Zeiss Axio Imager.A2 upright fluorescent microscope (Carl Zeiss Inc.) with a 10x objective and green fluorescent protein filter. Exposure levels were set using ATP treated samples and all subsequent images were taken with the same settings. Vacuoles were classified by relative intensity $(2=$ high intensity, $1=$ low intensity, $0=$ no intensity), and fluorescence intensity of each vacuole was quantified using Fiji software ${ }^{16}$. A circular region within the vacuole being measured was quantified, and average intensities for each sample were statistically compared.

Autophagy was quantified in seedling roots by staining of acidic organelles with the lipophilic dye MDC and in leaf protoplasts by transient expression of the autophagosome marker GFP-ATG8e as previously described ${ }^{56}$. Chemical complementation of the ski2-5 constitutive autophagy phenotype was performed as in Kazibwe et al. ${ }^{10}$, except that inosine was used at a final concentration of $50 \mu \mathrm{M}$. MDC-stained autophagosomes were counted per frame from at least 10 root images taken in the late elongation zone, while GFP-labeled structures were counted from at least 100 protoplasts with active autophagy. All experiments were done in triplicate per genotype.

Immunoblotting. ATG8 protein accumulation was analyzed as described previously, with minor modifications ${ }^{10}$. Briefly, seven-day old WT, ski2-5, rh40-1, rh40-2, ski2-5/SKI2 and $r n s 2-2$ seedlings were incubated with or without $50 \mu \mathrm{M}$ inosine (final concentration) in liquid $1 / 2 \mathrm{MS}$ medium supplemented or not with $1 \mu \mathrm{M}$ final concentration of concanamycin $\mathrm{A}$ (ConcA) for 12 hours, and total protein was separated by SDS-PAGE followed by immunoblotting with anti-ATG8a antibody (AS14 2769, Agrisera).

Statistical analysis. Except as indicated, all data were analyzed in triplicate using R (version 4.0.3). Twotailed Student's $t$-tests were computed for pairwise comparison of means of data sets, with the statistical significance level set to 0.05 . The reported $p$ values were adjusted to account for multiple comparisons. For all figures, error bars are the mean \pm SD. 


\section{Acknowledgements}

This work was supported by a grant from the National Science Foundation (MCB-1714996) to G.C.M. and D.C.B.

\section{Contributions}

B.E.F., G.C.M. and D.C.B. conceived the project and designed experiments. B.E.F., Z.K, S.C.M., Y.M., AY.L., V.R. and X.L. performed experiments and analyzed data. B.E.F., Z.K., G.C.M. and D.C.B. prepared the manuscript. All authors revised the manuscript.

\section{Extended data}

Extended Figure 1. Drosophila melanogaster rRNA-specific primers. Total RNA was extracted from Arabidopsis rosette leaves and Drosophila ovaries and tested by RT-PCR using primers against either Drosophila or Arabidopsis rRNA sequences. The Drosophila primers are specific for Drosophila $18 \mathrm{~S}$ and $28 \mathrm{~S}$ rRNA. The positions of size markers are shown at left. At: Arabidopsis thaliana, Dm: Drosophila melanogaster.

Extended Figure 2. Alexa488-tagged-RNA is transported into rns2-2 vacuoles in vitro. A) The number of rns2-2 vacuoles at zero, low, and high fluorescence intensity for three biological replicates was quantified and normalized to the total number of vacuoles. B) Fraction of low and high fluorescence vacuoles normalized to total number of fluorescent vacuoles. Error bars represent \pm SD. Similar letters indicate no significant difference according to two-way analysis of variance (ANOVA) with Tukey's multiple comparisons test; For (A), a, b; b, c; a, c; $p<0.0001$; For (B), a, b; b, c; b, e; $p<0.01, a, c ; a, e ; p$ $<0.0001, \mathrm{c}, \mathrm{e} ; \mathrm{p}<0.05$. FL, fluorescence.

Extended Figure 3. Characterization of the rh40-1, rh40-2 and ski2-5 mutant lines. A) Schematic of the two independent T-DNA insertion alleles of $r h 40$. B) RT-PCR was used to detect the presence of $R H 40$ transcripts. The lack of $R H 40$ transcripts in the mutant lines indicates that $r h 40-1$ and $r h 40-2$ are null mutants. C) Schematic of the T-DNA insertion allele of ski2-5. D) RT-PCR was used to detect the presence of SKI2 transcripts in the ski2-5 mutants. A reduced amount of transcript was observed in the mutant line. E). Q-PCR was used to quantify the relative amount of $S K I 2$ transcript present in the ski2-5 mutant. Results indicate that $S K I 2$ is expressed in the ski2-5 mutant at less than $1 \%$ of the wild type level. The ski2-5/SKI2 line corresponds to the ski2-5 mutant complemented with a wild type SKI2 gene, and shows expression similar to wild type. Error bars indicate \pm standard deviation from three biological replicates, with means for each replicate represented by the blue circle, black triangle and brown square. Similar letters on each column indicate no significant differences according to pairwise Student's two-sided $t$ test; $a, b, p<0.001$.

Extended Figure 4. ski2 and rh40 mutants have increased basal autophagy. Representative confocal microscope images of MDC-stained roots of ski2-5 (A), rh40-1 and rh40-2 (B) seedlings compared to wild type. rns2-2 and atg7-2 are used as controls for increased autophagy and reduced autophagy, respectively. Scalebar $=20 \mu \mathrm{m}$. C, D) MDC-labeled puncta as shown in parts (A) and (B) were counted from at least 15 late elongation zone root images per genotype with three biological replicates. Each replicate is color coded as blue circles, black triangles and brown squares. Larger horizontal bar indicates mean, error bars represent \pm SD. Similar letters indicate no significant difference according to pairwise Student's two-sided equal variance $t$-test. For (C), a, b $p<0.0001 ; \mathrm{a}, \mathrm{c} p<0.05$. For (D) a, b $p<0.001$. 
Extended Figure 5. Autophagy activation in ski2 but not $r h 40$ is blocked by inosine treatment. Quantification of MDC-stained structures in roots of seedlings of the indicated genotypes without inosine treatment, or after 3-hour incubation with $10 \mu \mathrm{M}(\mathrm{A})$ or $50 \mu \mathrm{M}(\mathrm{B})$ inosine (final concentration) in liquid $1 / 2 \mathrm{MS}$ medium. MDC-labelled puncta were counted from at least 10 root images taken in the late elongation zone per genotype with three biological replicates, with each replicate color coded in blue circles, black triangles and brown squares. Larger horizontal bar indicates mean, error bars represent \pm SD. Similar letters indicate no significant difference according to pairwise Student's twosided equal variance $t$-test. For (A), a, b $p<0.05$; for (B) a, b $p<0.001$.

Extended Figure 6. Quantification of the number of protoplasts from wild type, ski2-5, rh40-1, rh40-2 and $\boldsymbol{r n s 2 - 2}$ with active autophagy. Protoplasts transiently expressing GFP-ATG8e were incubated overnight in W5 solution, supplemented or not with inosine at $10 \mu \mathrm{M}(\mathrm{A})$ or $50 \mu \mathrm{M}$ (B) final concentration. Protoplasts with 3 or more GFP-labeled puncta were considered to have active autophagy and were normalized as a percentage of total protoplasts. The three biological replicates are color coded in blue circles, black triangles and brown squares. Larger horizontal bar indicates mean, error bars represent \pm SD. Similar letters indicate no significant difference according to pairwise Student's two-sided equal variance $t$-test. For $(A), a, b p<0.001$. For (B), a, b $p<0.05$.

Extended Figure 7. The rh40 autophagy phenotype is not affected by inosine treatment. A) Seven-day old wild type, rh40-1 and $r h 40-2$ seedlings were incubated with or without $50 \mu \mathrm{M}$ inosine (final concentration) in liquid $1 / 2 \mathrm{MS}$ medium, and treated or not with $1 \mu \mathrm{M}$ final concentration of concanamycin A (ConcA) for 12 hours. Proteins from crude extracts were separated by SDS-PAGE followed by immunoblotting with anti-ATG8 antibodies. Shown is a representative image from three independent biological replicates. B) Quantification of ATG8 band intensity, normalized to the Ponceau S-stained band corresponding to Rubisco large subunit, with the value of the wild type control set to 1. Larger horizontal bar indicates mean, error bars represent \pm SD for three biological replicates with means for each replicate indicated as blue circle, black rectangle and brown square. Similar letters indicate no significant difference according to pairwise Student's two-sided equal variance $t$-test. a, b $p<$ $0.05 ; \mathrm{a}, \mathrm{c} p<0.001 ; \mathrm{a}, \mathrm{d} p<0.0001 ; \mathrm{b}, \mathrm{c} p<0.05 ; \mathrm{b}, \mathrm{d} p<0.01 ; \mathrm{c}, \mathrm{d} p<0.05$.

\section{Supplementary data}

Supplementary Table 1. Primers used in this study

\section{Figure legends}

Figure 1. RNA is transported into plant vacuoles in a process that requires ATP. A) Schematic of the experimental design to measure the transport of Drosophila RNA (squiggles) into purified Arabidopsis vacuoles (circles). Vacuoles were incubated with RNA in the presence or absence of ATP. After incubation, unincorporated RNA was degraded with RNase A. RNA imported into vacuoles was protected from degradation, and was quantified using qRT-PCR. Vacuolar acid phosphatase activity was used for normalization. B) Quantification of RNA transport into rns2-2 vacuoles. RNA transport was analyzed with or without addition of ATP. Apyrase was used to remove residual ATP in the -ATP treatment. Abundance of $18 \mathrm{~S}$ and $28 \mathrm{~S}$ Drosophila rRNA incorporated into vacuoles was quantified by qRT-PCR, and the results were normalized to acid phosphatase activity. The results are expressed relative to the WT average. Samples were analyzed in triplicate for four biological replicates. Larger horizontal bar indicates mean, error bars represent \pm SD. The mean and SD were computed from the means of three biological replicates, indicated by blue square, brown triangle and black circle. Similar 
letters indicate no significant difference according to pairwise Student's two-sided $t$-test; $\mathrm{a}, \mathrm{b}$ and $\mathrm{a}, \mathrm{c} p$ $<0.0001 ; b, c p<0.05$.

Figure 2. Alexa488-tagged-RNA is transported into plant vacuoles in vitro. A) Schematic of experimental design for transport of fluorescently labeled RNA (in green) to purified vacuoles (circles). Vacuoles were incubated with FL-RNA in the presence or absence of ATP, diluted to reduce background fluorescence, and incorporation of RNA into vacuoles was determined by microscopy. B) Fluorescence microscopy and differential interference contrast (DIC) microscopy images of vacuole examples with zero, low, and high fluorescence (FL). Scalebar $=25 \mu \mathrm{m}$. C) Confocal microscopy z-series of $2.7 \mu \mathrm{m}$ optical sections of low (top panel) and high (bottom panel) fluorescing vacuoles. Scalebar $=20 \mu \mathrm{m}$. D) Quantification of the number of vacuoles with zero, low, and high fluorescence intensity for three biological replicates normalized to the total number of vacuoles. E) Fraction of low and high fluorescence vacuoles normalized to total number of fluorescent vacuoles. Error bars represent \pm SD. Similar letters indicate no significant difference according to Two-way analysis of variance (ANOVA) with Tukey's multiple comparisons test. For (D), a, b; b, c; $a, c p<0.0001$. For (E) $a, b ; b, c ; b$, e $p<0.01, a, c$; a, e $p<0.0001$, c, e $p<0.05$.

Figure 3. High fluorescence vacuoles are increased in the presence of ATP. FL-RNA transport was analyzed using vacuoles purified from wild type (A) or rns2-2 mutant (B) plants. Fluorescence intensity for each vacuole was determined from microscopy images using Fiji software. Each experiment was repeated three times. The number of vacuoles analyzed is indicated below each plot. Larger horizontal bar indicates mean, error bars represent \pm SD. The mean and SD were computed from the means of three biological replicates, indicated by blue triangle, brown square and black circle. Similar letters indicate no significant difference according to pairwise Student's two-sided $t$-test. For (A) and (B), $a, b p$ $<0.05 ; \mathrm{b}, \mathrm{c} p<0.001 ; \mathrm{a}, \mathrm{c} p<0.0001$.

Figure 4. ATP hydrolysis is necessary for efficient RNA transport into vacuoles. FL-RNA import into isolated vacuoles was determined by fluorescence quantification of microscopy images. A) Treatment of vacuoles with a non-hydrolysable ATP (adenosine $5^{\prime}$-( $\beta, \gamma$-imido) triphosphate lithium salt hydrate). B) Treatment of vacuoles with the ABC-transporter inhibitor sodium orthovanadate. C) Treatment of vacuoles with the ion gradient uncoupler gramicidin. Larger horizontal bar indicates mean, error bars represent $\pm S D$. The mean and SD were computed from the means of three (A) or two (B and $C$ ) biological replicates, indicated by blue circle, brown square and black triangle. The number of vacuoles analyzed is indicated below each plot. Similar letters indicate no significant difference according to pairwise Student's two-sided $t$-test, $\mathrm{a}, \mathrm{b} p<0.05$.

Figure 5. SKI2 is necessary for efficient RNA transport into vacuoles. A) FL-RNA transport into vacuoles isolated from wild type, ski2-5, and the ski2-5 line complemented with a wild type copy of the SKI2 gene (ski2-5/SKI2). B) FL-RNA transport into vacuoles isolated from wild type and the rh40-1 and $r h 40-2$ lines. The total number of vacuoles is indicated below each plot. Larger horizontal bar indicates mean, error bars represent \pm SD. The mean and SD were computed from the means of three biological replicates, indicated by blue circle, brown square and black triangle. Similar letters indicate no significant difference according to pairwise Student's two-sided $t$-test; $\mathrm{a}, \mathrm{b} p<0.05$.

Figure 6. SKI2 deficiency causes increased basal autophagy that is reduced by inosine treatment. A) Representative confocal microscopy images of protoplasts from wild type and ski2-5 lines transformed with a GFP-ATG8e marker. Scale bars $=20 \mu \mathrm{m}$, GFP, Green Fluorescent Protein, BF, Bright field. B) Quantification of the number of protoplasts from wild type, ski2-5, ski2-5/SKI2, and rns2-2 showing 
more than three GFP-positive puncta, normalized as percentage of total protoplasts. Autophagosomes (GFP-positive puncta) were counted from three biological replicates (blue circles, brown squares and black rectangles). Larger horizontal bar indicates mean, error bars represent \pm SD. Similar letters indicate no significant difference according to pairwise Student's two-sided equal variance $t$-test. $\mathrm{a}, \mathrm{b}$ and $\mathrm{a}, \mathrm{c} p<$ $0.001 ; b, c p<0.05$. C, D) Seven-day old seedlings of the indicated genotypes were incubated with or without $50 \mu \mathrm{M}$ inosine (final concentration) in liquid $1 / 2 \mathrm{MS}$ medium supplemented with or without $1 \mu \mathrm{M}$ final concentration of concanamycin A (ConcA) for 12 hours, and total protein was separated by SDSPAGE followed by immunoblotting with anti-ATG8 antibodies. Shown are representative images from three independent biological replicates. E, F) Quantification of the ATG8 band intensity, compared to the Ponceau S-stained band corresponding to Rubisco large subunit, and normalized using the wild type value. Larger horizontal bar indicates mean, error bars represent \pm SD for three biological replicates (blue circle, black rectangle and brown square). Similar letters indicate no significant difference according to pairwise Student's two-sided equal variance $t$-test. For both (E) and (F), a, b $p<0.05 ; \mathrm{a}, \mathrm{c} p$ $<0.001 ; \mathrm{a}, \mathrm{d} p<0.0001 ; \mathrm{b}, \mathrm{c} p<0.05 ; \mathrm{b}, \mathrm{d} p<0.01 ; \mathrm{c}, \mathrm{d} p<0.05$.

\section{References}

1 Ashihara, H., Stasolla, C., Fujimura, T. \& Crozier, A. Purine salvage in plants. Phytochemistry 147, 89-124, doi:https://doi.org/10.1016/i.phytochem.2017.12.008 (2018).

2 Marsac, R. et al. Purine Homeostasis Is Necessary for Developmental Timing, Germline Maintenance and Muscle Integrity in Caenorhabditis elegans. Genetics 211, 1297-1313, doi:10.1534/genetics.118.301062 (2019).

3 Garcia-Gil, M. et al. Emerging Role of Purine Metabolizing Enzymes in Brain Function and Tumors. International Journal of Molecular Sciences 19, 3598 (2018).

4 Moffatt, B. A. \& Ashihara, H. Purine and Pyrimidine Nucleotide Synthesis and Metabolism. The Arabidopsis Book 2002 (2002).

5 Witte, C.-P. \& Herde, M. Nucleotide Metabolism in Plants. Plant Physiology 182, 63-78, doi:10.1104/pp.19.00955 (2020).

6 Hillwig, M. S. et al. RNS2, a conserved member of the RNase T2 family, is necessary for ribosomal RNA decay in plants. Proc Natl Acad Sci U S A 108, 1093-1098 (2011).

7 Floyd, B. E., Mugume, Y., Morriss, S. C., Maclntosh, G. C. \& Bassham, D. C. Localization of RNS2 ribonuclease to the vacuole is required for its role in cellular homeostasis. Planta 245, 779-792, doi:10.1007/s00425-016-2644-x (2017).

8 Floyd, B. E., Morriss, S. C., MacIntosh, G. C. \& Bassham, D. C. Evidence for autophagy-dependent pathways of rRNA turnover in Arabidopsis. Autophagy 11, $2199-2212$ (2015). 
9 Morriss, S. C., Liu, X., Floyd, B. E., Bassham, D. C. \& Maclntosh, G. C. Cell growth and homeostasis are disrupted in arabidopsis rns2-2 mutants missing the main vacuolar RNase activity. Annals of Botany 120, 911-922, doi:10.1093/aob/mcx099 (2017).

10 Kazibwe, Z., Soto-Burgos, J., Maclntosh, G. C. \& Bassham, D. C. TOR mediates the autophagy response to altered nucleotide homeostasis in an RNase mutant. J Exp Bot 71, 6907-6920, doi:10.1093/jxb/eraa410 (2020).

11 Kraft, C., Deplazes, A., Sohrmann, M. \& Peter, M. Mature ribosomes are selectively degraded upon starvation by an autophagy pathway requiring the Ubp3p/Bre5p ubiquitin protease. Nature Cell Biology 10, 602-610, doi:ncb1723 [pii] 10.1038/ncb1723 (2008).

12 Fujiwara, Y. et al. Discovery of a novel type of autophagy targeting RNA. Autophagy 9, 403-409, doi:10.4161/auto.23002 (2013).

13 Aizawa, S. et al. Lysosomal putative RNA transporter SIDT2 mediates direct uptake of RNA by lysosomes. Autophagy 12, 565-578, doi:10.1080/15548627.2016.1145325 (2016).

14 Altschul, S. F., Gish, W., Miller, W., Myers, E. W. \& Lipman, D. J. Basic Local Alignment Search Tool. J Mol Biol 215, 403-410 (1990).

15 Goodstein, D. M. et al. Phytozome: a comparative platform for green plant genomics. Nucleic Acids Res. 40, D1178-D1186, doi:10.1093/nar/gkr944 (2011).

16 Schindelin, J. et al. Fiji: an open-source platform for biological-image analysis. Nature Methods 9, 676-682, doi:10.1038/nmeth.2019 (2012).

17 Kang, J. et al. Plant ABC Transporters. The Arabidopsis Book 2011 (2011).

18 Goodno, C. C. Inhibition of myosin ATPase by vanadate ion. Proceedings of the National Academy of Sciences 76, 2620-2624 (1979).

19 Etxeberria, E., Pozueta-Romero, J. \& Gonzalez, P. In and out of the plant storage vacuole. Plant Sci 190, 52-61, doi:https://doi.org/10.1016/j.plantsci.2012.03.010 (2012).

20 Karlish, S. J. D., Shavit, N. \& Avron, M. On the Mechanism of Uncoupling in Chloroplasts by lonPermeability Inducing Agents. European Journal of Biochemistry 9, 291-298, doi:10.1111/j.14321033.1969.tb00608.x (1969).

21 Andersen, O. S., Koeppe, R. E. \& Roux, B. Gramicidin channels. IEEE Transactions on NanoBioscience 4, 10-20, doi:10.1109/TNB.2004.842470 (2005).

22 Carter, C. et al. The Vegetative Vacuole Proteome of Arabidopsis thaliana Reveals Predicted and Unexpected Proteins. Plant Cell 16, 3285-3303, doi:10.1105/tpc.104.027078 (2004).

23 Zhao, L. \& Kunst, L. SUPERKILLER Complex Components Are Required for the RNA ExosomeMediated Control of Cuticular Wax Biosynthesis in Arabidopsis Inflorescence Stems. Plant Physiology 171, 960-973, doi:10.1104/pp.16.00450 (2016). 
24 Lange, H. et al. RST1 and RIPR connect the cytosolic RNA exosome to the Ski complex in Arabidopsis. Nature Communications 10, 3871, doi:10.1038/s41467-019-11807-4 (2019).

25 Palm, D. et al. Plant-specific ribosome biogenesis factors in Arabidopsis thaliana with essential function in rRNA processing. Nucleic Acids Res. 47, 1880-1895, doi:10.1093/nar/gky1261 (2019).

26 Boudet, N., Aubourg, S., Toffano-Nioche, C., Kreis, M. \& Lecharny, A. Evolution of Intron/Exon Structure of DEAD Helicase Family Genes in Arabidopsis, Caenorhabditis, and Drosophila. Genome Res 11, 2101-2114, doi:10.1101/gr.200801 (2001).

27 Klionsky, D. J. et al. Guidelines for the use and interpretation of assays for monitoring autophagy (4th edition)1. Autophagy 17, 1-382, doi:10.1080/15548627.2020.1797280 (2021).

28 Bao, Y. et al. COST1 regulates autophagy to control plant drought tolerance. Proceedings of the National Academy of Sciences 117, 7482-7493, doi:10.1073/pnas.1918539117 (2020).

29 James, A., Wang, Y., Raje, H., Rosby, R. \& DiMario, P. Nucleolar stress with and without p53. Nucleus 5, 402-426, doi:10.4161/nucl.32235 (2014).

30 Kazibwe, Z., Liu, A.-Y., MacIntosh, G. C. \& Bassham, D. C. The Ins and Outs of Autophagic Ribosome Turnover. Cells 8, 1603 (2019).

31 Taylor, C. B., Bariola, P. A., Delcardayre, S. B., Raines, R. T. \& Green, P. J. RNS2 - a SenescenceAssociated RNase of Arabidopsis That Diverged from the S-RNases before Speciation. P Natl Acad Sci USA 90, 5118-5122 (1993).

32 Hase, K. et al. RNautophagy/DNautophagy possesses selectivity for RNA/DNA substrates. Nucleic Acids Res. 43, 6439-6449, doi:10.1093/nar/gkv579 (2015).

33 Liu, H. et al. Skeletal muscle-specific Sidt2 knockout in mice induced muscular dystrophy-like phenotype. Metabolism - Clinical and Experimental 85, 259-270, doi:10.1016/j.metabol.2018.05.004 (2018).

34 Hase, K. et al. Cytosolic domain of SIDT2 carries an arginine-rich motif that binds to RNA/DNA and is important for the direct transport of nucleic acids into lysosomes. Autophagy 16, 19741988, doi:10.1080/15548627.2020.1712109 (2020).

35 Cuervo, A. M. \& Dice, J. F. Unique properties of lamp2a compared to other lamp2 isoforms. Journal of Cell Science 113, 4441-4450 (2000).

36 Fujiwara, Y., Hase, K., Wada, K. \& Kabuta, T. An RNautophagy/DNautophagy receptor, LAMP2C, possesses an arginine-rich motif that mediates RNA/DNA-binding. Biochemical and Biophysical Research Communications 460, 281-286, doi:https://doi.org/10.1016/j.bbrc.2015.03.025 (2015).

37 Schuck, S. Microautophagy - distinct molecular mechanisms handle cargoes of many sizes. Journal of Cell Science 133, doi:10.1242/jcs.246322 (2020).

38 Sieńko, K., Poormassalehgoo, A., Yamada, K. \& Goto-Yamada, S. Microautophagy in Plants: Consideration of Its Molecular Mechanism. Cells 9, 887 (2020). 
39 Chekanova, J. A. et al. Genome-Wide High-Resolution Mapping of Exosome Substrates Reveals Hidden Features in the Arabidopsis Transcriptome. Cell 131, 1340-1353, doi:10.1016/j.cell.2007.10.056 (2007).

40 Mitchell, P. \& Tollervey, D. An NMD Pathway in Yeast Involving Accelerated Deadenylation and Exosome-Mediated 3'->5' Degradation. Molecular Cell 11, 1405-1413, doi:10.1016/S10972765(03)00190-4 (2003).

41 Mühlemann, O. \& Lykke-Andersen, J. How and where are nonsense mRNAs degraded in mammalian cells? RNA Biology 7, 28-32, doi:10.4161/rna.7.1.10578 (2010).

42 Szádeczky-Kardoss, I., Gál, L., Auber, A., Taller, J. \& Silhavy, D. The No-go decay system degrades plant mRNAs that contain a long A-stretch in the coding region. Plant Sci 275, 19-27, doi:https://doi.org/10.1016/j.plantsci.2018.07.008 (2018).

43 Szádeczky-Kardoss, l. et al. The nonstop decay and the RNA silencing systems operate cooperatively in plants. Nucleic Acids Res. 46, 4632-4648, doi:10.1093/nar/gky279 (2018).

44 Limoncelli, K. A., Merrikh, C. N. \& Moore, M. J. ASC1 and RPS3: new actors in 18S nonfunctional rRNA decay. RNA 23, 1946-1960, doi:10.1261/rna.061671.117 (2017).

45 Yatsuka, H. et al. Exosc2 deficiency leads to developmental disorders by causing a nucleotide pool imbalance in zebrafish. Biochemical and Biophysical Research Communications 533, 1470 1476, doi:https://doi.org/10.1016/j.bbrc.2020.10.044 (2020).

46 Zhang, X. et al. Suppression of endogenous gene silencing by bidirectional cytoplasmic RNA decay in Arabidopsis. Science 348, 120-123, doi:10.1126/science.aaa2618 (2015).

47 Branscheid, A. et al. SKI2 mediates degradation of RISC 5 '-cleavage fragments and prevents secondary siRNA production from miRNA targets in Arabidopsis. Nucleic Acids Res. 43, 1097510988, doi:10.1093/nar/gkv1014 (2015).

$48 \mathrm{Li}$, T. et al. A genetics screen highlights emerging roles for CPL3, RST1 and URT1 in RNA metabolism and silencing. Nature Plants 5, 539-550, doi:10.1038/s41477-019-0419-7 (2019).

49 Hooker, T. S., Lam, P., Zheng, H. \& Kunst, L. A Core Subunit of the RNA-Processing/Degrading Exosome Specifically Influences Cuticular Wax Biosynthesis in Arabidopsis. The Plant Cell 19, 904-913, doi:10.1105/tpc.106.049304 (2007).

50 Yang, X. et al. CER16 Inhibits Post-Transcriptional Gene Silencing of CER3 to Regulate Alkane Biosynthesis. Plant Physiology 182, 1211-1221, doi:10.1104/pp.19.01002 (2020).

51 Liu, Y. et al. Degradation of the endoplasmic reticulum by autophagy during endoplasmic reticulum stress in Arabidopsis. The Plant Cell 24, 4635-4651, doi:10.1105/tpc.112.101535 (2012).

52 Robert, S., Zouhar, J., Carter, C. \& Raikhel, N. Isolation of intact vacuoles from Arabidopsis rosette leaf-derived protoplasts. Nat. Protocols 2, 259-262 (2007). 
53 Ambrosio, L. et al. Phylogenetic analyses and characterization of RNase X25 from Drosophila melanogaster suggest a conserved housekeeping role and additional functions for RNase T2 enzymes in protostomes. PLoS One 9, e105444, doi:10.1371/journal.pone.0105444 (2014).

54 Ahmed, S. U. et al. The Plant Vacuolar Sorting Receptor Atelp Is Involved in Transport of Nh2Terminal Propeptide-Containing Vacuolar Proteins in Arabidopsis thaliana. The Journal of Cell Biology 149, 1335-1344, doi:10.1083/jcb.149.7.1335 (2000).

55 Reilly, T. J., Baron, G. S., Nano, F. E. \& Kuhlenschmidt, M. S. Characterization and Sequencing of a Respiratory Burst-inhibiting Acid Phosphatase from Francisella tularensis. J Biol Chem 271, 10973-10983, doi:10.1074/jbc.271.18.10973 (1996).

56 Contento, A. L., Xiong, Y. \& Bassham, D. C. Visualization of autophagy in Arabidopsis using the fluorescent dye monodansylcadaverine and a GFP-AtATG8e fusion protein. Plant J 42, 598-608, doi:TPJ2396 [pii]10.1111/j.1365-313X.2005.02396.x (2005). 


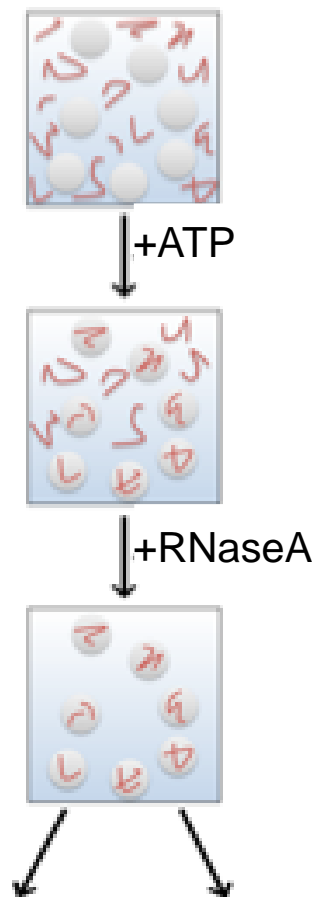

RNA Extraction Acid Phosphatase qRT-PCR Activity Analysis

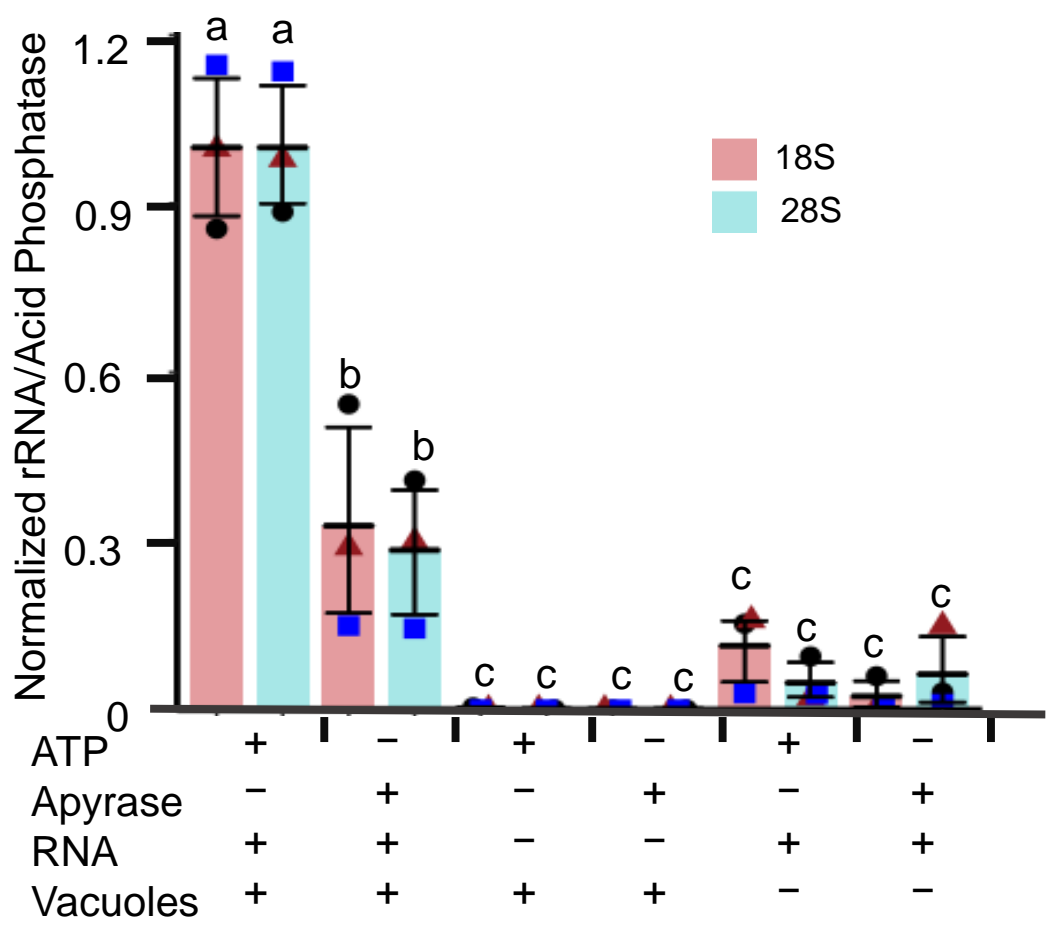

Figure 1. RNA is transported into plant vacuoles in a process that requires ATP. A) Schematic of the experimental design to measure the transport of Drosophila RNA (squiggles) into purified Arabidopsis vacuoles (circles). Vacuoles were incubated with RNA in the presence or absence of ATP. After incubation, unincorporated RNA was degraded with RNase A. RNA imported into vacuoles was protected from degradation, and was quantified using qRT-PCR. Vacuolar acid phosphatase activity was used for normalization. B) Quantification of RNA transport into rns2-2 vacuoles. RNA transport was analyzed with or without addition of ATP. Apyrase was used to remove residual ATP in the -ATP treatment. Abundance of $18 \mathrm{~S}$ and $28 \mathrm{~S}$ Drosophila rRNA incorporated into vacuoles was quantified by qRT-PCR, and the results were normalized to acid phosphatase activity. The results are expressed relative to the WT average. Samples were analyzed in triplicate for four biological replicates. Larger horizontal bar indicates mean, error bars represent \pm SD. The mean and SD were computed from the means of three biological replicates, indicated by blue square, brown triangle and black circle. Similar letters indicate no significant difference according to pairwise Student's two-sided t-test; $a, b$ and $a, c p<0.0001 ; b, c p<0.05$. 


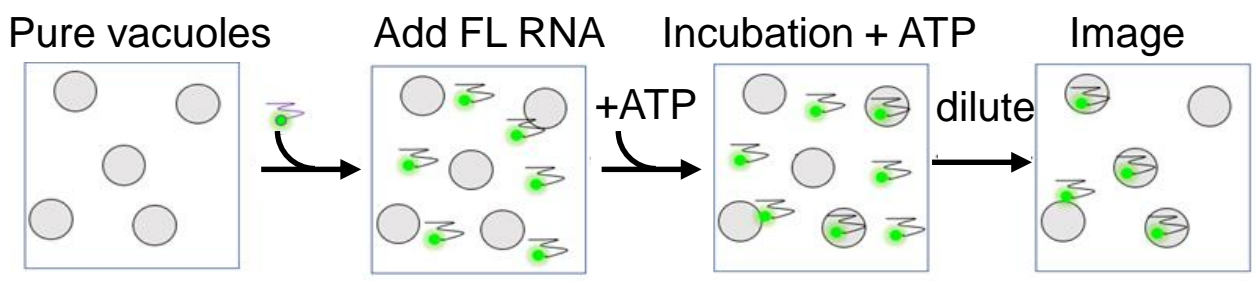

B

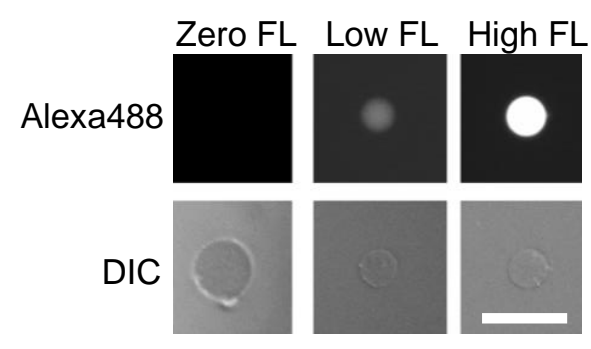

D

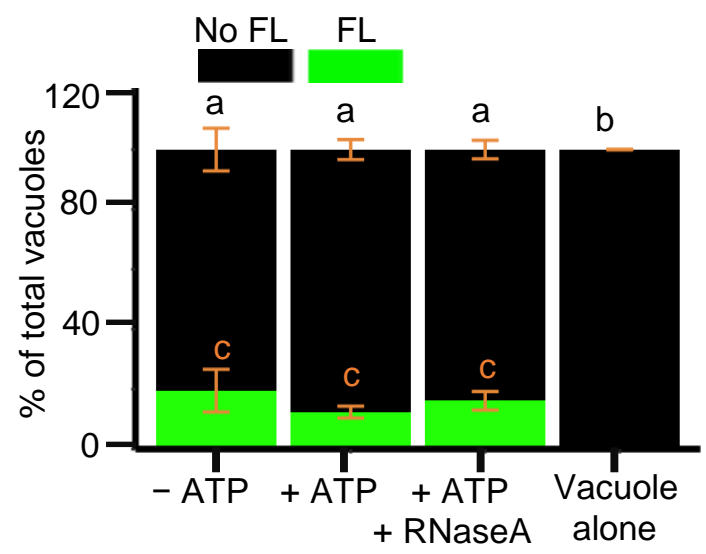

C
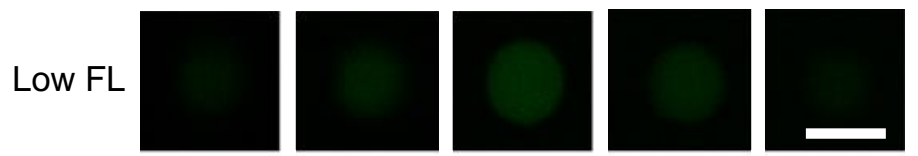

High FL
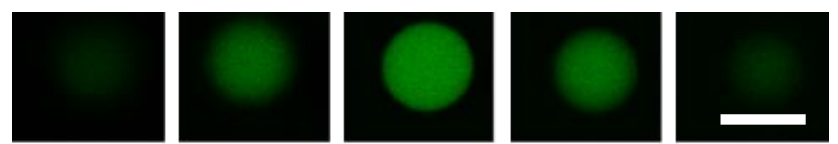

E

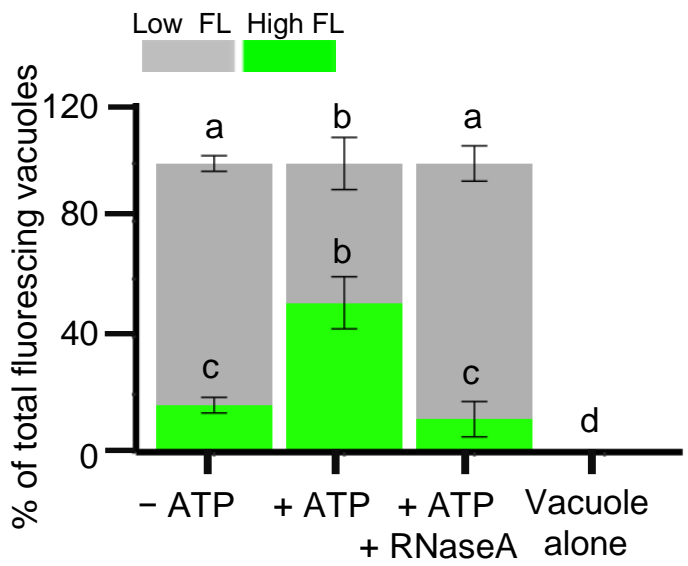

Figure 2. Alexa488-tagged-RNA is transported into plant vacuoles in vitro. A) Schematic of experimental design for transport of fluorescently labeled RNA (in green) to purified vacuoles (circles). Vacuoles were incubated with FL-RNA in the presence or absence of ATP, diluted to reduce background fluorescence, and incorporation of RNA into vacuoles was determined by microscopy. B) Fluorescence microscopy and differential interference contrast (DIC) microscopy images of vacuole examples with zero, low, and high fluorescence (FL). Scalebar $=25 \mu \mathrm{m}$. C) Confocal microscopy z-series of $2.7 \mu \mathrm{m}$ optical sections of low (top panel) and high (bottom panel) fluorescing vacuoles. Scalebar = $20 \mu \mathrm{m}$. D) Quantification of the number of vacuoles with zero, low, and high fluorescence intensity for three biological replicates normalized to the total number of vacuoles. E) Fraction of low and high fluorescence vacuoles normalized to total number of fluorescent vacuoles. Error bars represent \pm SD. Similar letters indicate no significant difference according to Two-way analysis of variance (ANOVA) with Tukey's multiple comparisons test. For (D), a, b; b, c; $a, c p<0.0001$. For (E) $a, b ; b, c ; b, e p<$ 0.01 , a, c; a, e $p<0.0001$, c, e $p<0.05$. 

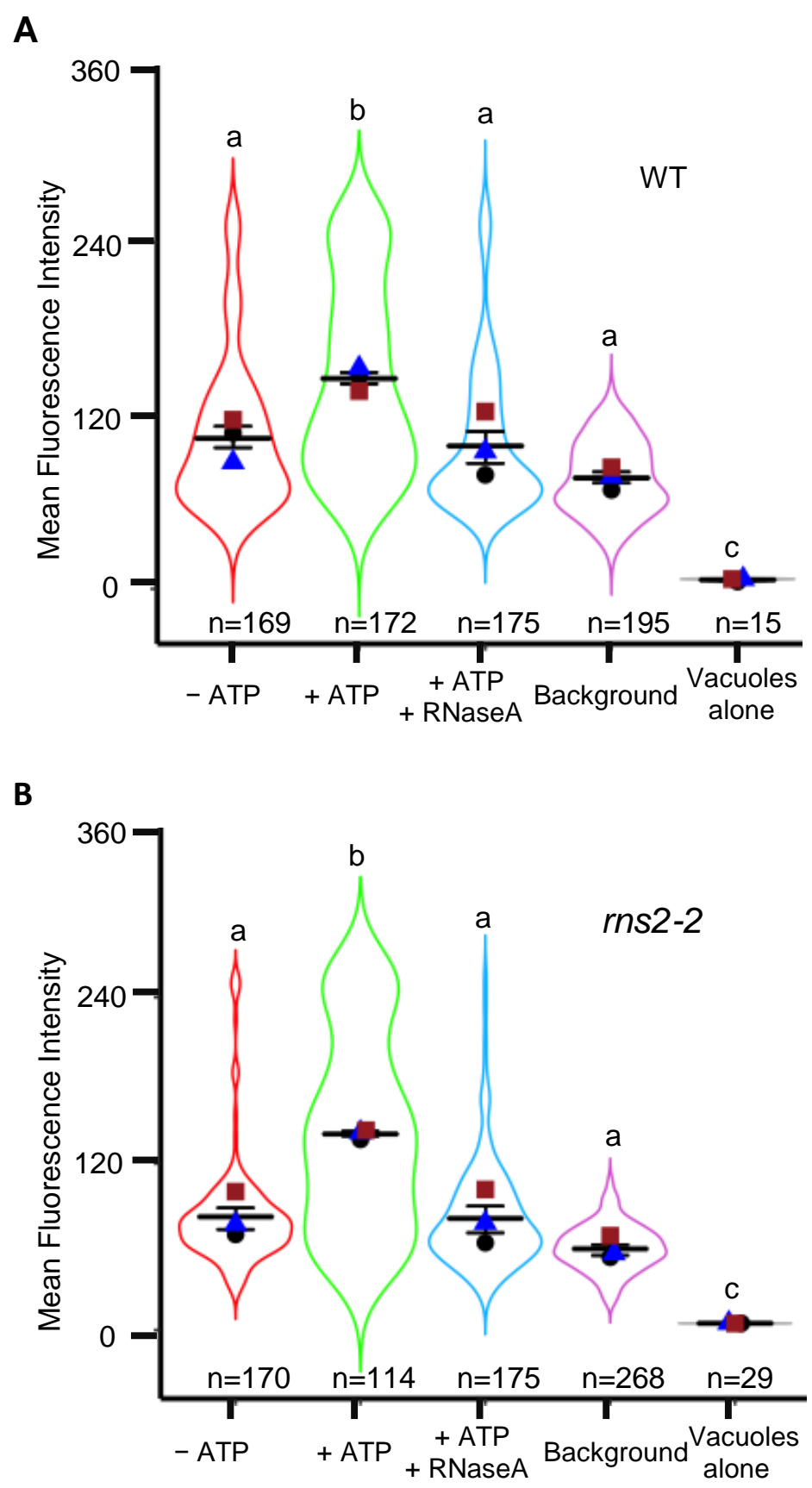

Figure 3. High fluorescence vacuoles are increased in the presence of ATP. FL-RNA transport was analyzed using vacuoles purified from wild type (A) or rns2-2 mutant (B) plants. Fluorescence intensity for each vacuole was determined from microscopy images using Fiji software. Each experiment was repeated three times. The number of vacuoles analyzed is indicated below each plot. Larger horizontal bar indicates mean, error bars represent \pm SD. The mean and SD were computed from the means of three biological replicates, indicated by blue triangle, brown square and black circle. Similar letters indicate no significant difference according to pairwise Student's two-sided $t$-test. For (A) and (B), a, b $p<0.05 ; \mathrm{b}, \mathrm{c} p<0.001 ; \mathrm{a}, \mathrm{c} p<0.0001$. 
A

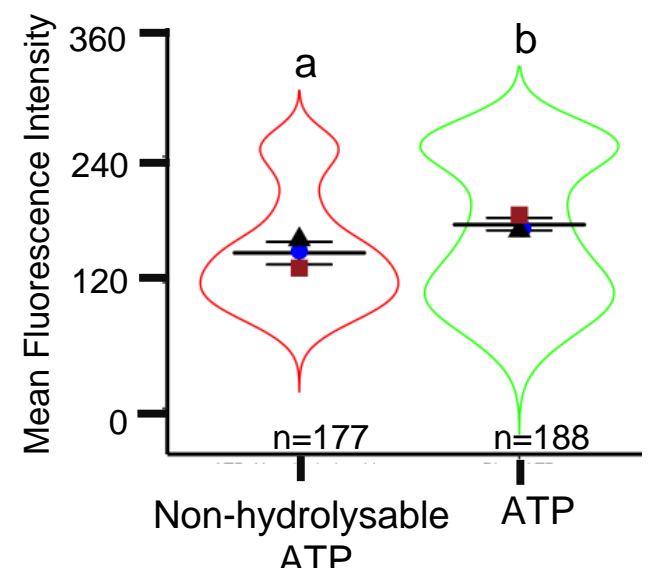

B

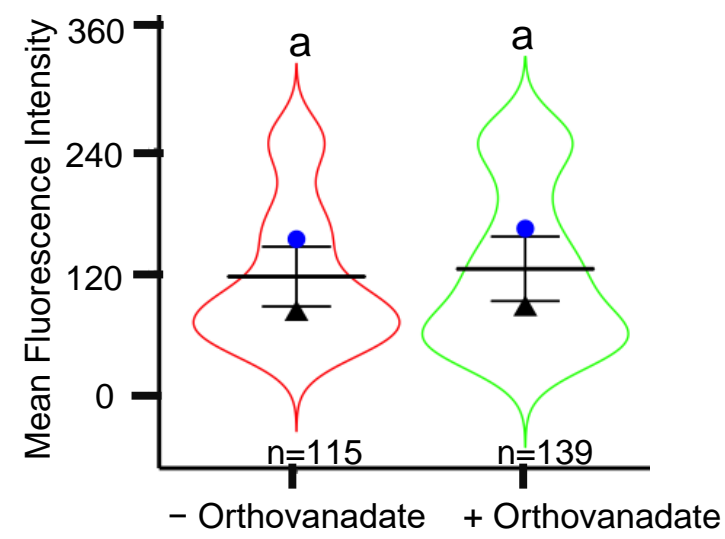

C

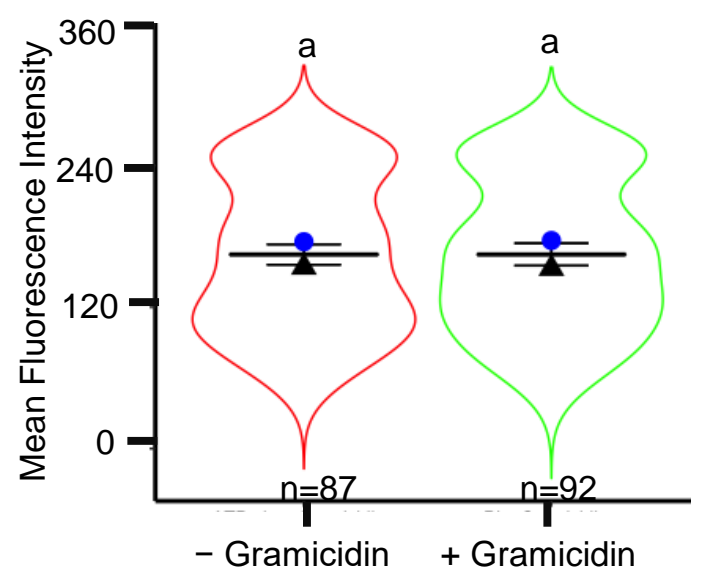


Figure 4. ATP hydrolysis is necessary for efficient RNA transport into vacuoles. FL-RNA import into isolated vacuoles was determined by fluorescence quantification of microscopy images. A) Treatment of vacuoles with a non-hydrolysable ATP (adenosine $5^{\prime}$ - $(\beta, \gamma$-imido) triphosphate lithium salt hydrate). B) Treatment of vacuoles with the $A B C$-transporter inhibitor sodium orthovanadate. C) Treatment of vacuoles with the ion gradient uncoupler gramicidin. Larger horizontal bar indicates mean, error bars represent \pm SD. The mean and SD were computed from the means of three $(A)$ or two ( $B$ and $C$ ) biological replicates, indicated by blue circle, brown square and black triangle. The number of vacuoles analyzed is indicated below each plot. Similar letters indicate no significant difference according to pairwise Student's two-sided $t$-test, $\mathrm{a}, \mathrm{b} p<0.05$. 


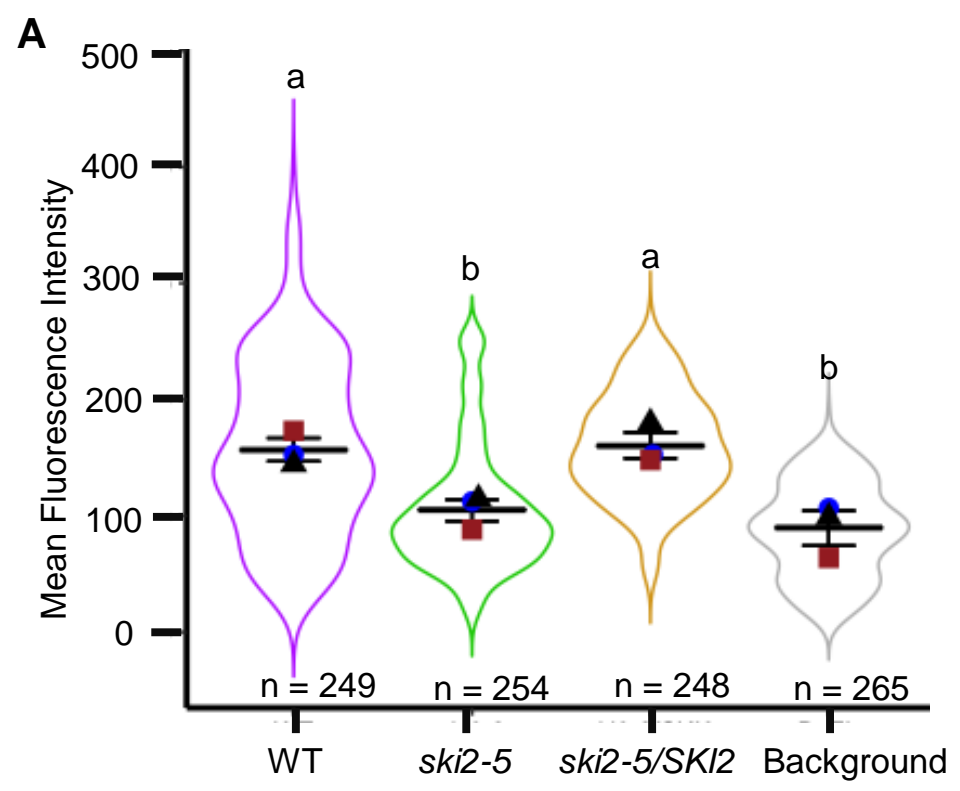

B

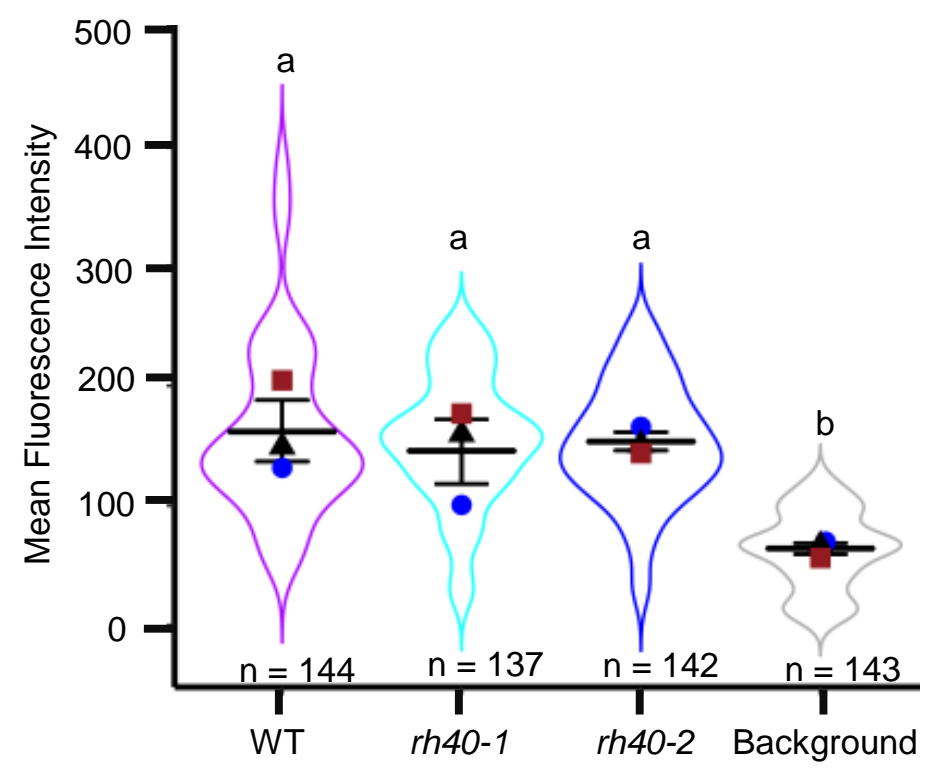

Figure 5. SKI2 is necessary for efficient RNA transport into vacuoles. A) FL-RNA transport into vacuoles isolated from wild type, ski2-5, and the ski2-5 line complemented with a wild type copy of the $S K I 2$ gene (ski2-5/SKI2). B) FL-RNA transport into vacuoles isolated from wild type and the $r h 40-1$ and $r h 40-2$ lines. The total number of vacuoles is indicated below each plot. Larger horizontal bar indicates mean, error bars represent \pm SD. The mean and SD were computed from the means of three biological replicates, indicated by blue circle, brown square and black triangle. Similar letters indicate no significant difference according to pairwise Student's two-sided $t$-test; $\mathrm{a}, \mathrm{b} p<0.05$. 
A

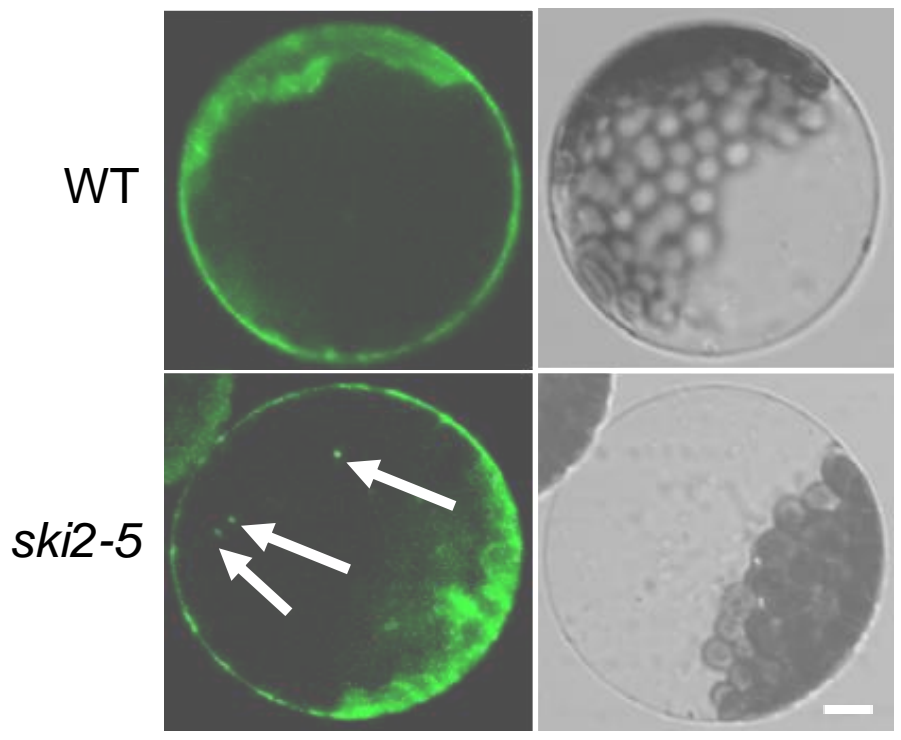

C

E
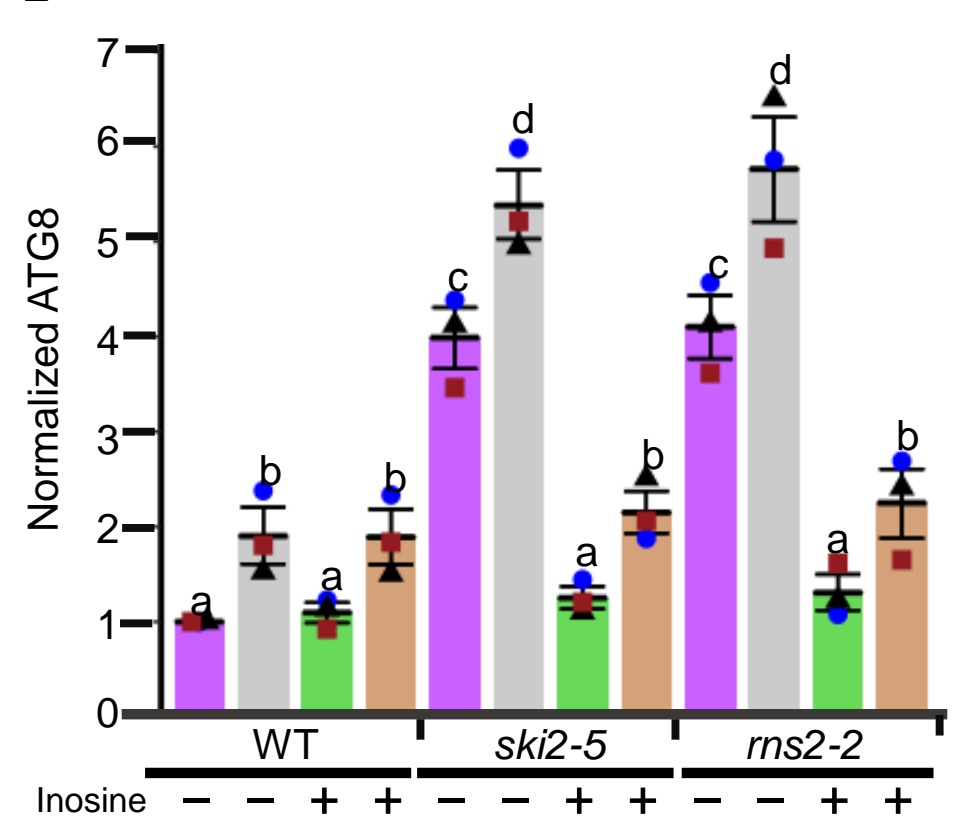

ConcA -+-+-+-+-+-+
B

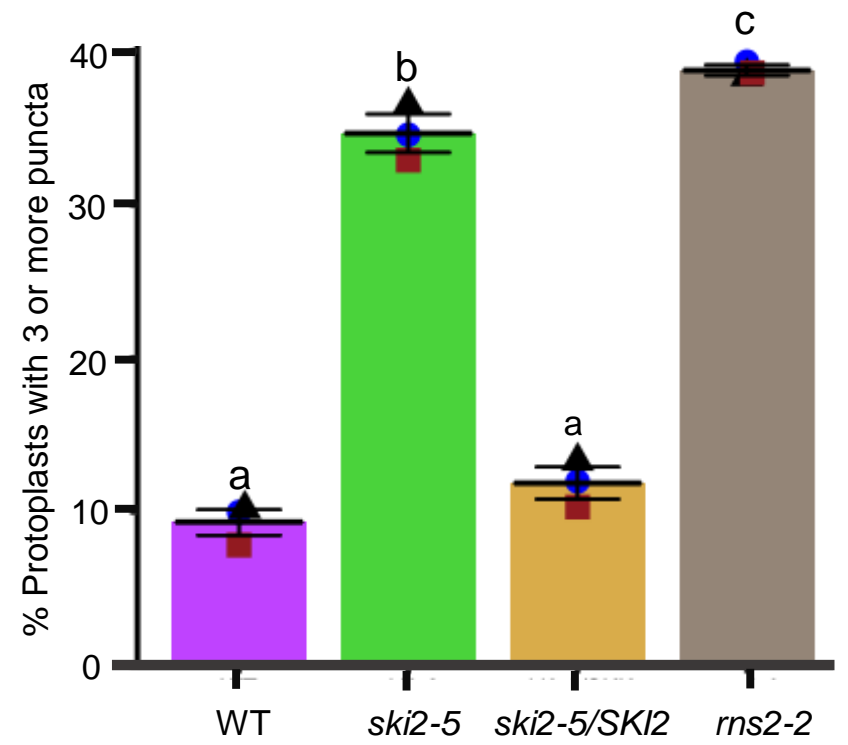

D $\frac{\text { WT }}{\text { snosine }--++-5 i 2-5} \frac{\text { ski2-5/SKI2 }}{-++++++}$

ConcA $-+-++-++-t_{\mathrm{kDa}}$ $-17$

Ponceau

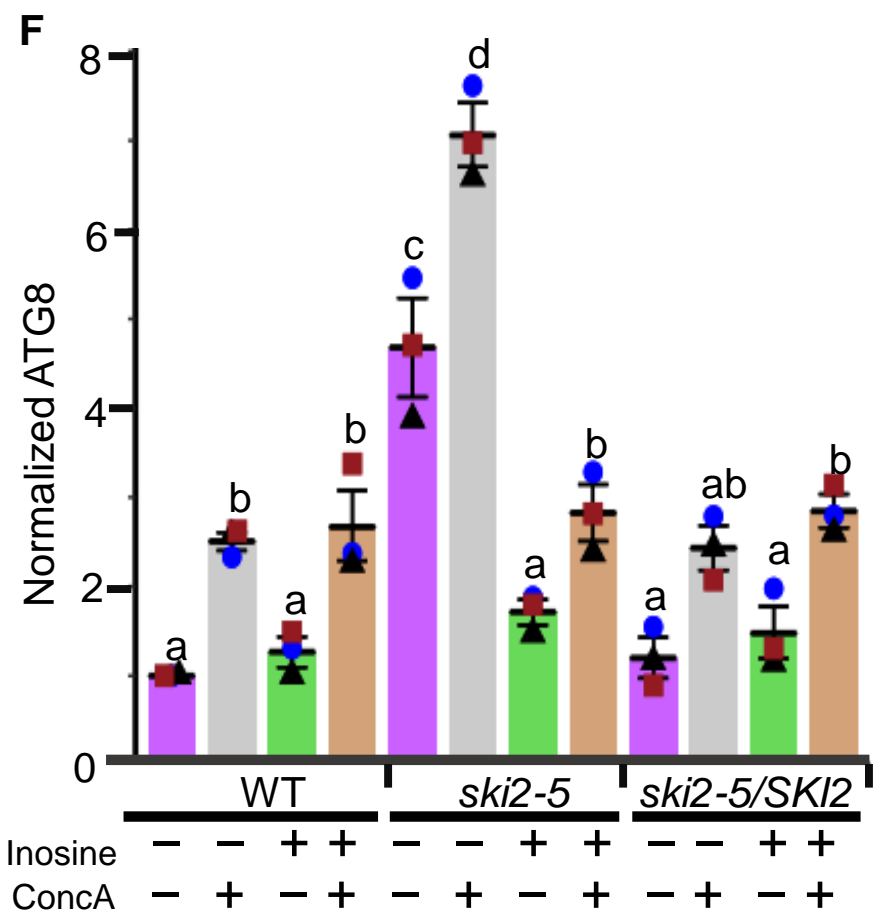


Figure 6. SKI2 deficiency causes increased basal autophagy that is reduced by inosine treatment. A) Representative confocal microscopy images of protoplasts from wild type and ski2-5 lines transformed with a GFP-ATG8e marker. Scale bars = $20 \mu \mathrm{m}, \mathrm{GFP}$, Green Fluorescent Protein, BF, Bright field. B) Quantification of the number of protoplasts from wild type, ski2-5, ski2-5/SKI2, and rns2-2 showing more than three GFP-positive puncta, normalized as percentage of total protoplasts. Autophagosomes (GFP-positive puncta) were counted from three biological replicates (blue circles, brown squares and black rectangles). Larger horizontal bar indicates mean, error bars represent \pm SD. Similar letters indicate no significant difference according to pairwise Student's two-sided equal variance $t$-test. $\mathrm{a}, \mathrm{b}$ and a, $c p<0.001 ; b, c p<0.05$. C, D) Seven-day old seedlings of the indicated genotypes were incubated with or without $50 \mu \mathrm{M}$ inosine (final concentration) in liquid $1 / 2 \mathrm{MS}$ medium supplemented with or without $1 \mu \mathrm{M}$ final concentration of concanamycin A (ConcA) for 12 hours, and total protein was separated by SDS-PAGE followed by immunoblotting with anti-ATG8 antibodies. Shown are representative images from three independent biological replicates. E, F) Quantification of the ATG8 band intensity, compared to the Ponceau S-stained band corresponding to Rubisco large subunit, and normalized using the wild type value. Larger horizontal bar indicates mean, error bars represent \pm SD for three biological replicates (blue circle, black rectangle and brown square). Similar letters indicate no significant difference according to pairwise Student's two-sided equal variance $t$-test. For both $(\mathbf{E})$ and $(\mathbf{F})$, a, b $p<0.05 ; \mathrm{a}, \mathrm{c} p<0.001$; a, d $p<0.0001 ; \mathrm{b}, \mathrm{c} p<0.05 ; \mathrm{b}, \mathrm{d} p<0.01 ; \mathrm{c}, \mathrm{d} p<0.05$. 